\title{
ORGANISATION SCIENTIFIQUE DU TRAVAIL DANS UNE EXPLOITATION APICOLE PROFESSIONNELLE EN PARTICULIER À L'AIDE DE LA TECHNIQUE D'ORDONNANCEMENT
}

\section{INTRODUCTION}

Les travaux relatifs à l'organisation scientifique du travail en apiculture sont peu nombreux quoique cette branche de l'agriculture soit susceptible d'offrir un large champ d'activités aux spécialistes; en effet, les méthodes de travail apicoles sont, au moins en Allemagne, susceptibles d'une nette amélioration.

Parmi les quelques auteurs qui se sont efforcés jusqu'ici de faire une analyse du travail apicole, je voudrais citer Piel-Desruisseaux qui a étudié le processus d'extraction du miel et comparé différentes possibilités d'utilisation des machines et du personnel, ainsi que Kassparian qui a fait l'analyse de la méthode de conduite des ruches de Hohenheim et qui a utilisé la technique d'ordonnancement et établi des plans de travail pour petite et moyenne exploitations.

Mes recherches ont été conduites également pour une part importante à l'aide de la technique d'ordonnancement; elles ne concernent pas une petite ou une moyenne exploitation mais l'entreprise professionnelle de la firme MAck.

\section{OBJECTIFS ET MÉTHODES}

Les objectifs des recherches étaient : égalisation des charges de travail, abaissement de la quantité de travail requise et augmentation du produit dans

* Traduction J. Louveaux. 
les exploitations apicoles professionnelles et, en particulier, dans celle de la firme Mack à Illertissen en Bavière. Pour atteindre ces objectifs j'ai tout d'abord étudié la situation effective de l'exploitation du point de vue de l'organisation et des techniques de travail et j'ai essayé, à l'aide de la technique d'ordonnancement, d'obtenir une égalisation des charges de travail. Ensuite, des propositions d'amélioration ont été élaborées et, sur cette base, quatre modes d'exploitation nouveaux ont été développés.

\section{Étude de la situation effective de l'exploitation du point de vue de l'organisation et des techniques de travail}

Au moment où les recherches ont été faites il y avait sur l'exploitation 6 apiculteurs et 2 apprentis occupés à plein temps. Pour les « greffages » de larves et pour l'extraction du miel on dispose selon les besoins du moment de 1 à 4 autres ouvriers à plein temps ou à mi-temps. On travaille avec des ruches du modèle Zander à 9 cadres. Un second corps de ruche est utilisé comme hausse. Comme appareillage on dispose de deux extracteurs électriques à 6 cadres réversibles, d'une étuve pour le couvain et d'un véhicule.

On effectue 3 à 5 transhumances avec les 1000 colonies de production. En été elles sont groupées par 48 et en hiver elles sont rassemblées dans de grands ruchers près d'Illertissen. Les colonies sont visitées 11 fois de façon intensive. On pratique le contrôle de l'essaimage, principalement par déplacement des cadres de couvain, par changement des reines tous les deux ans et par formation d'essaims artificiels.

D'autre part, on élève environ 5000 reines en trois séries, aussi bien pour les besoins internes que pour la vente. Comme colonies éleveuses on utilise environ 145 colonies d'abeilles des bruyères. Les $2 / 5$ des jeunes reines sont amenées dans des nuclei à un cadre vers des stations de fécondation spéciales. Les $3 / 5$ sont vendues comme reines vierges.

Au cours des années 1967 et 1968 j’ai étudié les travaux qui doivent être faits à terme, c'est-à-dire ceux qui ne peuvent être effectués que pendant l'été, selon les jours du calendrier, selon la nature du travail et selon les produits. L'étude selon les jours du calendrier met en évidence une forte pointe de travail en mai, juin et juillet et une autre, plus faible, à la fin d'août et au début de septembre. L'étude selon la nature du travail montre que les travaux les plus importants sont les visites, les transhumances et les extractions de miel qui, de ce fait, justifient particulièrement une rationalisation. L'étude selon les produits des travaux qui doivent être faits à terme montre qu'il faut par an, 16 minutes pour $1 \mathrm{~kg}$ de miel et respectivement 5 heures 17 minutes et 30,3 minutes pour une colonie et pour une reine féconde. 
Un chiffre intéressant apparaît lorsqu'on confronte le nombre d'heures de travail exigées au revenu en DM; le revenu - miel et reines - par heure de travail a été en moyenne de 15,01 DM en 1967 et 1968.

\section{Utilisation de la technique d'ordonnancement pour l'égalisation des charges de travail}

La situation effective ayant été déterminée on procéda sur elle à des recherches. On utilisa tout d'abord la technique d'ordonnancement avec pour objectif l'égalisation des charges de travail c'est-à-dire le déplacement des pointes de travail vers les creux. Un plan d'ordonnancement est une espèce particulière de planning à l'aide duquel on peut, entre autre, déterminer de combien est la marge de temps pour un travail. J'ai établi un plan d'ordonnancement pour le semestre d'été et j'ai essayé au moyen d'un tableau de planning de décaler les travaux dans le cadre de la marge de temps de telle sorte que les pointes de travail et les creux s'égalisent plus ou moins. Le nombre des heures supplémentaires n'a pu toutefois être abaissé que de $17 \%$. Ceci m'amena à penser qu'il fallait élargir la marge de temps en modifiant la conduite de l'exploitation et obtenir de cette façon une plus forte égalisation. Pour cela également la technique d'ordonnancement est un moyen approprié car dans le plan d'ordonnancement on peut voir clairement les dépendances qui limitent les marges de temps et par conséquent les possibilités de décalage des activités.

L'élevage des reines, le nourrissement d'hivernage et la transhumance de retour à l'automne apparaissent comme particulièrement intéressants pour le décalage; par exemple, en avançant les élevages de reines on pourrait contribuer à faire disparaître les pointes de travail estivales et à combler les creux du printemps. Cependant, dans la forme actuelle, il n'est pas possible d'avancer les élevages de reines car au moment des " greffages » de larves il est nécessaire de connaître les colonies raceuses ce qui signifie qu'il faut que le travail de contrôle de la sélection soit achevé. On pourrait faire la proposition de modification suivante : si on n'utilise pour la première série d'élevage que les colonies les plus âgées qui ont déjà été contrôlées l'année précédente, on peut alors avancer l'élevage sous la forme d'une pré-série jusqu'à l'obstacle suivant - la date la plus précoce de maturité des mâles — sqit d'environ 17 jours.

En ce qui concerne le nourrissement d'automne, il est effectué au rucher d'hivernage et constitue de ce fait l'obstacle décisif au recul de la transhumance de retour qui lui-même conditionne l'étalement des tâches. On peut faire la proposition suivante : si aussitôt après l'enlèvement des hausses et avant la transhumance, on nourrit en utilisant des nourrisseurs de grande capacité de telle sorte qu'on ne soit pas obligé d'opérer en plusieurs fois, la transhumance de retour devient indépendante du nourrissement d'automne et plus librement disponible. 
Développement, calcul et représentation de 4 nouveaux modes de conduite

Sur la base des données acquises par les recherches j'ai développé 4 nouveaux modes de conduite avec pour préoccupation dominante de réaliser une technique de conduite des ruches aussi extensive que possible, c'est-à-dire exigeant moins de temps. Dans cet esprit j'ai pris particulièrement en considération les trois faits suivants : $1^{\circ}$ les colonies qui ont une reine d'un an essaiment rarement; $2^{\circ}$ les colonies qui ont assez de place pour le couvain et le miel essaiment moins que les colonies qui sont à l'étroit; $3^{\circ}$ les colonies de force moyenne essaiment moins que les faibles et les très fortes.

Compte tenu de ces faits, on pourrait réduire le nombre des visites sans que les pertes d'essaims soient plus grandes et se limiter au cours des différentes visites aux prélèvements de miel et aux nourrissements. Ceci fut déjà en partie réalisé dans un premier projet.

1. Mode d'exploitation intensive-extensive combinée, selon un projet de Hans Schlüter Jr.

La différence fondamentale par rapport aux modes d'exploitation utilisés jusqu'ici est que pour un effectif égal de 240 colonies on travaille de façon plus extensive que jusqu'à présent. La conduite est plus extensive en ce sens que le nombre des visites est plus faible (les visites $2,4,5,7$ et 8 sont supprimées) et que les temps de travail sur les colonies sont en partie raccourcis; cependant ces temps sont, en partie, plus longs. En général on se contente de nourrir ou on récolte du miel. Le changement de reine continue à se produire pour toutes les colonies selon un cycle de 2 ans ce qui fait que les colonies conduites de façon extensive ont des reines qui n'ont qu'un an.

La formation d'essaims artificiels est limitée avec les colonies conduites de façon extensive car elles sont en été sur 3 corps et en hiver sur 2 corps avec 15 cadres. L'installation des 240 colonies conduites de façon extensive ne se fait plus par groupes de $\mathbf{4 8}$ mais seulement de 30 à chaque emplacement de transhumance. Ainsi le nombre de ces emplacements est augmenté de 3 .

La répartition des charges de travail ne présente aucune amélioration et, la somme de travail, qu'une amélioration faible par rapport aux méthodes habituelles. On n'a pu calculer qu'une diminution de 71 heures supplémentaires. Celles-ci passent de 880 à 809. (Les heures supplémentaires concernent les 6 ouvriers faisant chacun 40 heures normales par semaine.)

Par colonie, le travail qui doit être fait à terme est de 5 heures et 14 minutes contre 5 heures et 17 minutes pour les méthodes de conduite habituclles. La quantité de travail par reine reste inchangée. 
Une augmentation du rendement en miel peut intervenir d'une part du fait qu'il y a moins de colonies par emplacement et, d'autre part, du fait qu'on a des colonies plus fortes (colonies sur 3 corps). On calcule par heure de travail un revenu de 15,10 DM contre 15,01 DM par les méthodes habituelles.

L'expérience réalisée en 1968 d'un travail extensif sur 16 colonies à 3 corps a donné pour ces 16 colonies un rendement moyen de $53 \mathrm{~kg}$. Le rendement moyen des colonies à 2 corps conduites de manière intensive a été pour les mêmes emplacements de transhumance de $32 \mathrm{~kg}$.

Pour éviter toute confusion signalons que l'on qualifie d'extensive la méthode qui consiste à limiter les opérations de contrôle de l'essaimage qui exigent beaucoup de temps. Les nourrissements de compensation et les nourrissements spéculatifs continuent à être pratiqués. C'est pourquoi des pertes de rendement ne pourraient provenir que de la perte d'essaims naturels. Des 16 colonies travaillées de façon extensive, aucune n'a essaimé, à ma connaissance. Seule une colonie a été perdue; elle est devenue bourdonneuse. La perte d'une colonie entre juillet et octobre sur 16 colonies se tient dans les mêmes limites que pour les colonies conduites de façon intensive. Dans la même période une colonie sur 20 a été dissoute parmi celles qui ont été conduites de façon intensive. Il est vrai que les colonies conduites de façon extensive avaient toutes des reines d'un an.

Cette méthode de conduite intensive-extensive combinée a été mise en application en 1970 sous une forme quelque peu différente. On a installé 200 colonies avec des reines $d$ 'un an de race pure d'élevage selon la manière habituelle par groupes de 48 à 50 unités. Pour contrôler l'essaimage on s'est contenté de mettre début mai à mi-mai un $3^{e}$ corps de ruche sous la grille à reine entre le nid à couvain et le magasin à miel. Ces colonies permettaient pour la première fois l'utilisation de la pleine miellée de pissenlit dans l'Allgäu, sans essaimage artificiel. On s'est limité dans les travaux à quelques sondages et à l'enlèvement du miel. Fin juillet début août on a fait une transhumance sur une miellée de sapin relativement courte. Ce fut malgré tout une réussite totale. On a ainsi à nouveau fait la preuve que des colonies de force convenable sont aussi capables en un temps relativement court de donner les plus fortes récoltes.

On peut encore ajouter que grâce à l'agrandissement généreux de la ruche au bon moment on peut renoncer à un contrôle régulier lorsque le matériel est impeccable du point de vue racial et lorsque les reines sont jeunes.

Pour le cas où l'on se déciderait à ne plus utiliser qu'une conduite extensive des ruches on a développé, calculé et représenté graphiquement une méthode de conduite purement extensive avec environ 1000 ruches et 3 séries d'élevages. 
2. Méthode d'exploitation extensive avec environ 1000 ruches et 3 séries d'élevage

La caractéristique de cette méthode consiste dans le fait que, sans modifier l'effectif des colonies, toutes les colonies de production sont conduites de façon plus extensive que jusqu'ici. Par méthode plus extensive il faut comprendre ici que, comme dans la méthode combinée intensive-extensive, on effectue moins de visites (les visites $2,4,5,7$ et 8 sont supprimées) et que les temps de travail sur chaque colonie sont en partie raccourcis et en partie rallongés. Le rallongement des temps de travail provient d'une visite d'été unique mais approfondie au cours de laquelle on procède à la confection d'un nombre un peu plus réduit d'essaims artificiels et au remplacement de toutes les reines des colonies de production selon un cycle d'un an.

Par ailleurs, la période pendant laquelle les colonies sont resserrées dure plus longtemps du fait que l'opération de réduction de l'espace disponible est conduite, elle aussi, plus à fond. Les colonies sont maintenues en été sur 3 corps et 27 cadres, en hiver sur deux corps et 15 cadres. L'installation des colonies de production ne se fait plus par groupes de 48 mais par groupes de 30 par emplacement de transhumance. Le nombre de ces emplacements se trouve ainsi augmenté de 10. Les colonies sont groupées par 5 dans des habitacles résistant aux conditions hivernales sur des supports communs avec un espace d'une demi-largeur de ruche entre elles. Elles restent tout l'hiver à l'extérieur au rucher d'hivernage.

A côté des visites les opérations de transhumance et d'extraction sont les travaux qui demandent le plus de temps. Pour arriver à une rationalisation aussi poussée que possible on a étudié, surtout sur ces opérations, des améliorations techniques. On suppose que les hausses sont manipulées au moyen de palettes, de chariots transporteurs de palettes, de tapis roulants ou de rouleaux, que l'on désopercule au moyen de machines à désoperculer, que l'on extrait au moyen de 3 extracteurs radiaires de 50 cadres et que le miel est envoyé directement dans les maturateurs par des tuyaux à la sortie de l'extracteur.

Pour les transhumances on suppose qu'on charge les ruches 5 par 5 dvec leur support sur le camion au moyen d'un appareil de levage hydraulique.

La répartition des charges de travail et particulièrement le niveau du travail exigé présentent une image nettement plus favorable. Au lieu de, respectivement 880 et 809 , on descend à 343 heures supplémentaires.

Le travail devant être exécuté à terme par colonie et par an n'est plus que de 3 heures et 38 minutes.

L'augmentation du rendement se poursuit du fait que tous les ruchers de transhumance sont occupés par un plus petit nombre de colonies et que toutes les ruches sont mises sur 3 corps. On arrive à 15,73 DM par heure de travail. 
Mais cette méthode de conduite des ruches ne constitue pas forcément un aboutissement. Par des mesures de rationalisation supplémentaires et en abandonnant l'élevage des reines l'effectif des colonies pourrait être encore fortement augmenté ainsi que le montre le calcul d'une nouvelle méthode de conduite extensive.

3. Méthode d'exploitation extensive avec environ 4000 colonies sans élevag̣e de reines.

Dans cette méthode d'exploitation le programme de production est modifié de façon fondamentale. On ne conserve que les colonies de production de miel. Les reines nécessaires ne sont pas élevées mais elles sont achetées à l'extérieur. Les essaims artificiels ne sont produits que pour la couverture des besoins de l'exploitation et non pas pour la vente. Les méthodes de production, qui dans ce cas ne concernent que le miel, sont en grande partie les mêmes que dans le cas d'une exploitation extensive de 1000 ruches. La différence la plus importante provient du fait qu'au lieu de ramener les ruches à l'automne sur les emplacements d'hiver on les conduit aux emplacements de transhumance de printemps. De cette façon la première transhumance de printemps devient superflue. Les colonies hivernent aux emplacements de printemps et non à la maison.

Du fait de l'hivernage sur les emplacements de printemps les différences suivantes interviennent : les travaux de printemps de même que les nourrissements d'automne n'ont plus lieu au rucher d'hiver mais aux emplacements de transhumance. Pour les travaux de la fin de l'été et de l'automne on a admis qu'à l'enlèvement des hausses de miel les colonies viennent déjà à leur emplacement d'hivernage et qu'on y entreprend le nourrissement d'automne, celui-ci étant fait avec des nourrisseurs de grande capacité qui n'ont pas besoin d'être rechargés plusieurs fois. L'enlèvement des nourrisseurs et la découverture des colonies doivent être réalisés en même temps au moment d'une visite de l'emplacement de transhumance.

La charge de travail est assez bien égalisée en ce qui concerne la surveillance des colonies. Ce résultat a été obtenu par décalage et rationalisation d'activités demandant beaucoup de travail. Par contre les travaux d'extraction provoquent des pointes de travail sensibles. Alors que le nombre des heures supplémentaires sans extraction n'atteint que 244 il monte avec l'extraction à l'énorme valeur de 1727 heures. L'utilisation de main-d'œuvre supplémentaire pour l'extraction s'impose, comme dans les autres cas.

La quantité de travail nécessaire par colonie et par an a pu être encore abaissée à 2 heures et 29 minutes. En mettant en service un second véhicule utilitaire on pourrait encore abaisser le temps nécessaire pour soigner les colo- 
nies. On ne ferait plus une équipe de 6 hommes mais deux équipes de 3 hommes. Par heure de travail le revenu atteint est de 42,63 DM.

A fin de montrer une exploitation qui pourrait livrer les reines nécessaires à son fonctionnement on a calculé la quantité de travail intervenant dans une méthode d'exploitation dont la dominante serait l'élevage des reines.

4. Méthode d'exploitation orientée sur l'élevage avec environ 200 colonies de production de race pure pour le choix des redroducteurs et 6 séries d'élevages avec environ 310 colonies éleveuses.

Les colonies de production et les colonies éleveuses sont travaillées de façon intensive comme dans la méthode actuelle. L'élevage des reines ne subit aucune transformation en ce qui concerne la division en séries d'élevage et la conduite des différentes séries. La différence essentielle réside :

$1^{\circ}$ dans une avance de l'élevage de reines,

$2^{\circ}$ dans un raccourcissement de l'espace de temps entre les points de départ des différentes séries, celui-ci étant ramené de 3 à 2 semaines et

$3^{\circ}$ dans le doublement du nombre des séries d'élevage qui passe de 3 à 6 .

L'avance de l'élevage de reines est obtenu de la manière suivante : dans la première série on utilise exclusivement des colonies d'élevage qui ont déjà été contrôlées l'année précédente et, par ailleurs, on emmène les colonies éleveuses pour cette série dans la vallée du Rhin de façon à garantir un développement rapide des colonies et une acceptation de nombreuses cellules royales dès la fin d'avril. De même on accélère le développement des mâles au moyen de mesures appropriées, par exemple, là aussi, par une transhumance dans la vallée du Rhin. Parallèlement le contrôle de sélection pour la seconde série est avancé.

Les travaux allant de l'orphelinage des colonies éleveuses jusqu'à la vente des reines et au nettoyage des ruchettes de fécondation a été planifié de telle sorte que les opérations se répètent tous les 14 jours et, dans toute la mesure du possible le même jour de la semaine. Ainsi, au bout d'un certain temps les ouvriers savent ce qu'ils ont à faire chaque jour sans avoir besoin de consulter le plan. Autant que possible le mercredi est libre de travaux d'élevage afin de pouvoir régler ce jour là l'essentiel des opérations de conduite des ruches.

La charge de travail est très favorable dans les mois de juin et de juillet. Les ouvriers sont presque complètement à jour pour un nombre relativement faible d'heures supplémentaires. Il n'y a en tout que 381 heures supplémentaires. Au cours des mois de mars, avril, août, septembre et octobre la capacité de travail existante n'est utilisée en gros que pour un tiers, de façon régulière, pour les travaux devant être exécutés à terme. 
Par la diminution de l'effectif des colonies de production jusqu'au minimum indispensable à l'élevage, non seulement la plupart des pointes de travail ont pu être éliminées mais encore la quantité de travail nécessaire a pu être diminuée d'une façon générale. Le temps de travail devant être exécuté à terme pour produire une reine vierge destinée à la vente est de 9,8 minutes contre 10,9 minutes avec les méthodes utilisées jusqu'ici. Pour une reine féconde de consommation le temps est de 25,9 minutes contre 30,3 par les méthodes habituelles.

La production s'élève grâce aux 3 séries supplémentaires avec chacune 55 colonies éleveuses de 5200 reines dans les conditions habituelles à environ 10900 reines. Par heure de travail le revenu est de 14,76 DM (miel et reines).

Une nouvelle augmentation, soit par élévation de la production par série, soit par multiplication des séries d'élevage au-delà de 6 - par exemple en passant à une série par semaine - est rendue impossible avec les méthodes habituelles par le fait que les capacités de travail sont limitées. Il faudra s'adresser à des méthodes qui épargnent le travail, par exemple la méthode du starter-finisseur. Ce dernier procédé ne pourra toutefois se révéler favorable que si l'on réussit à introduire sans grosses pertes dans les nuclei de fécondation les reines vierges écloses et marquées.

\section{DISCUSSION ET CONCLUSION}

Quelle méthode d'exploitation est finalement la meilleure?

La méthode extensive avec 4000 colonies représente la meilleure méthode d'exploitation si l'on prend comme critères le revenu brut de 42,63 DM par heure de travail, les 2 heures et 29 minutes de travail devant être effectué à terme par colonie et par an et les 244 heures supplémentaires, non compris l'extraction. Cette méthode d'exploitation n'est cependant pas réalisable à l'heure actuelle en raison des possibilités limitées d'achats de reines. Pour le moment il est donc nécessaire de continuer à produire du miel et à élever des reines.

La comparaison des différentes charges de travail montre que, aussi bien la méthode extensive avec 4000 colonies que la méthode orientée vers l'élevage ont des exigences équilibrées. Ce n'est pas le cas avec les deux autres méthodes qui sont une combinaison d'élevage de reines et de production de miel, activités qui sont rarement en harmonie. L'éleveur travaille selon un plan rigoureux tandis que le producteur de miel doit s'adapter constamment aux conditions atmosphériques et prendre ses dispositions au jour le jour. Si on ne dispose pas de plusieurs ouvriers l'une ou l'autre des productions est négligée.

Cependant, comme plusieurs ouvriers sont disponibles, on peut se repré- 
senter une méthode de conduite des ruches dans laquelle l'apiculteur peut profiter des avantages des deux méthodes spécialisées et faire de l'élevage tout en produisant du miel. Dans cette méthode d'exploitation l'élevage de reines et la production de miel doivent être séparés, aussi bien en ce qui concerne le personnel que le matériel d'exploitation. Cette séparation peut se faire de la façon suivante : une partie des ouvriers, par exemple deux, s'occupent exclusivement des élevages de reines, au moins pendant la saison principale, l'autre partie, par exemple 4 ouvriers, s'occupent des ruches. La séparation des personnels est importante car elle permet à chacun de se concentrer sur une tâche sans être dérangé; elle permet en outre de réaliser une production de masse ou une fabrication à la chaîne lesquelles exigent une forte spécialisation, de la concentration et une exécution à l'abri des dérangements.

Pour celà, en ce qui concerne la production du miel, il convient de donner aux travaux d'été une forme plus extensive de façon à éliminer les pointes de travail estivales et à obtenir un niveau de charge qui corresponde à celui du printemps et de l'automne. En bloquant ou en réduisant les colonies il devient possible d'utiliser régulièrement la capacité de travail existante de façon complète.

L'élevage des reines devrait se faire de façon continue, selon le principe de la fabrication à la chaîne, de façon à répartir régulièrement le travail des ouvriers chargés de cet élevage. Cela signifie qu'on ne devrait plus travailler sur un nombre réduit de grandes séries mais au contraire en faire beaucoup de plus petites, si possible avec la méthode du starter-finisseur, avec une charge de travail plus régulière.

Reçu pour publication en novembre 1973.

\section{LITERATURVERZEICHNIS}

Bittrer A., 1958. Über die Verteilung der Arbeiten in der Bienenzucht. Leipz. Bienen-Ztg. 72 (11), 316-320.

Bötтcher F. K., 1963. Bienenzucht als Erweb. Ein Handbuch für den wirtschaftlich arbeitenden Imker. 2. Aufl., München.

Brandenberger J. und Konrad R., 1965. Netzplantechnik. Eine Einführung. Schriftenreihe des Betriebswissenschaftlichen Institutes der ETH Zürich, Band 1.

Braukmany S., 1968. Kniffe und Winke auf dem Bienenstand. Zusammengestellt aus dem Vortrag des Imkermeisters Schlüter aus Illertissen. Nordwestd. Ztg. 20 (8), 207-212.

Condes W., 1965. 33 Jahre imkern mit der Großraumbeute. Nordwesid. I. Ztg. 17 (5), 115.

Erblmeier G., 1965. Erfolgreiche Bienenzucht in gemischter Waldtracht. Ein Imker-Lehrbuch für die Praxis. 1. Aufl., Simbach/Inn.

Eickmeyer K. A., 1963. Bienenwohnungsfragen (IV). Nordwestd. I. Ztg. 15 (4), 75-79.

Eickмeyer K. A., 1966. Zusammenhänge zwischen Beutengröße und Vollksstärke. Nordwestd. I. Ztg. 18 (1), 5-11.

EıскмеуеR K. A., 1967. Ist Wirtschaftlichkeit in der Imkerei nötig und erwünscht oder geht es auch ohne Wirtschaftlichkeit? Nordwestd. I. Ztg. 19 (2), 31-34. 
Еiскмеуев K. A., 1967. Ist. wirtschaftliche Imkerei möglich? Nordwestd. I. Ztg. 19 (3), 59-62.

v. Falkenhausen H., 1965. Prinzipien und Rechenverfahren der Netzplantechnik. ADLSchriftenreihe, Band 2, Kiel.

Forster H., 1971. Arbeitsanalyse eines Haupterwerbs-Imkereibetriebes, insbesondere mit Hilfe der Netzplantechnik. Diss. Universität Hohenheim.

Heiland H., Jandel A. und Kastrer W., 1966. Zur Anwendung der Netzplantechnik in der landwirtschaftlichen Unternehmensforschung. Agrarwirtschaft 15, 89-101.

Hesselbach J., 1960. Arbeitsaufwand in der Bienenhaltung. Der Imkerfreund 15 (10), 308312.

IBM, 1969. PCS Project Control System 1401. IMB Form 80 741-o.

Kassparian S., 1968. Arbeitswirtschaftliche Analyse der Hohenheimer imkerlichen Betriebsweise und Möglichkeiten mit Hilfe der Netzplantechnik. Z. Bienenforsch. 9 (7), 269-331.

Lock yеr K. G., 1968. Einführung in die Netzplantechnik. Autorisierte Übersetzung : Rationalisierungs-Kuratorium der Deutschen Wirtschaft e.V., Frankfurt/Main, Köln-Braunsfeld.

Owens Ch. D., und Detroy B. F., 1968. Neue technische Entwicklungen in der Bienenzucht. Apiacta 3, 4-14.

Piel-Desruisseaux J., 1965. Organisation du travail d'extraction du miel. Ann. Abeille 8 (3), 205-263.

Philippi M., 1933. Die wirtschaftliche Lage der deutschen Imkerei im Jahre 1931. Arch. Bienenkunde 14, 241-296.

REFA Arbeitsgemeinschaft der Verbände für Arbeitsstudien, 1967. Das REFA-Buch Band 1, Arbeitsgestaltung. Mit einer Einführung in das Arbeitsstudium. 10 Auf., Carl Hanser Verlag, München.

Sснмutz H., 1966. Die Netzwerktechnik. Siebte Jahrestagung der Gesellschaft für Wirtschaftsund Sozialwissenschaften des Landbaues e.V., Kiel.

Schundau W., 1969. Die Schwarmzeit. Nordwestd. I. Ztg. 21 (5), 123-125.

Steche W., 1968. Wie ernte ich mehr Honig? Der Imkerfreund 23 (2), 40-46.

StöcкмanN K., 1965. Wirtschaftliche Imkerei. Nordwestd. I. Ztg. 17 (12), 277-281.

Teske W., 1968. Besuch in der größten Imkerei der Welt, Miel Carlotta, Cuernavaca/Mexico. Nordwestd. I. Ztg. 20 (1), 14-16.

Townsend G. F., 1967. Bericht der Kommission für Technologie und Bienenzuchtgeräte, Apiacta 2, 40.42.

WILD E., 1969. Rentabilitätserhebungen und Buchhaltungswesen des VDSB. Schweiz. Bienen. Ztg. 92, 70-71. 